\title{
A radio continuum study of the Magellanic Clouds. IVa. Catalogue of radio sources in the Large Magellanic Cloud at $2.30 \mathrm{GHz}(\lambda=13 \mathrm{~cm})^{\star}$
}

\author{
M.D. Filipović ${ }^{1,2}$, G.L. White ${ }^{1}$, R.F. Haynes $^{2,1}$, P.A. Jones ${ }^{1}$, D. Meinert ${ }^{3}$, R. Wielebinski ${ }^{4}$ and U. Klein ${ }^{5}$ \\ ${ }^{1}$ University of Western Sydney, Nepean, P.O. Box 10, Kingswood, NSW 2747, Australia \\ 2 Australia Telescope National Facility, CSIRO, P.O. Box 76, Epping, NSW 2121, Australia \\ ${ }^{3}$ Institut für Atmosphärenphysik, Max-Planck-Straße, D-21502 Geesthacht-Tesperhude, Germany \\ ${ }^{4}$ Max-Planck-Institut für Radioastronomie, Auf dem Hügel 69, D-53121 Bonn, Germany \\ ${ }^{5}$ Radioastronomisches Institut der Universitat Bonn, Auf dem Hügel 71, D-53121 Bonn, Germany
}

Received January 12; accepted March 26, 1996

\begin{abstract}
We present a new catalogue of radio sources in the Large Magellanic Cloud (LMC) based on observations at $2.30 \mathrm{GHz}$ with the Parkes radio telescope. A total of 119 sources have been detected. We compare positions and flux densities of these sources with previously published radio results and find no significant positional displacement or flux discrepancies.
\end{abstract}

Key words: galaxies: Magellanic Clouds — radio continuum: galaxies — catalogue

\section{Introduction}

The most recent, published, large-scale and sensitive radio surveys of the LMC were undertaken at 1.40, 2.45, 4.75, $4.85,8.55 \mathrm{GHz}$ between 1988 and 1991 by a team from the Australia Telescope National Facility (ATNF) in Sydney, Australia, and the Max-Planck-Institut für Radioastronomie (MPIfR) in Bonn, Germany, using the Parkes $64-m$ radio telescope (Haynes et al. 1986, 1991). The catalogues of radio sources at these frequencies were published in Filipović et al. (1995) (hereafter Paper IV).

As part of this large-scale project, we observed the $\mathrm{LMC}$ at $2.30 \mathrm{GHz}$ and present here a catalogue of all discrete sources. We address issues related to the calibration of the survey: the source positional errors and the flux density calibration. The astrophysical interpretation of sources found in the surveys will be presented in later papers (Filipović et al. 1996a, b). Although close in frequency to the published data at $2.45 \mathrm{GHz}$ (Paper IV), the flux densities determined at $2.30 \mathrm{GHz}$ will give an extra datum in the source radio spectra and hence will increase our confidence in the estimate of the spectral index.

Send offprint requests to: M.D. Filipović

* Tables 1 and 2 are only available electronically at the CDS via ftp 130.79.128.5 or cdsarc.u-strasbg.fr

\section{The $2.30 \mathrm{GHz}$ survey}

The survey was made between 1987 November and 1988 January using the Parkes $64-m$ telescope fitted with the Australia Telescope National Facility prototype receiver package (Haynes et al. 1986, 1991). Observations were made by scanning the telescope across the LMC in right ascension and declination at driving rates in the range $1^{\circ}$ to $4^{\circ}$ per minute. The beam size of the $2.30 \mathrm{GHz}$ survey is $8.85^{\prime}$. We based our work on a field size defined by the $2.45 \mathrm{GHz}$ survey field from Paper IV, namely the region $\sim 10^{\circ}$ by $10^{\circ}$ centred on RA $(1950)=05^{\mathrm{h}} 30^{\mathrm{m}} 00^{\mathrm{s}}$ and Dec $(1950)=-68^{\circ} 30^{\prime} 00^{\prime \prime}$. The rms noise of this survey is estimated to be $30 \pm 5 \mathrm{mJy}^{\text {beam }}{ }^{-1}$. Figure 1 is a map of the LMC at $2.30 \mathrm{GHz}$.

Calibration was undertaken by frequently observing sources with known flux density and position and by mapping them in the same way as for the LMC. The main source for flux density calibration was Hydra A for which the flux density was assumed to be $26.3 \mathrm{Jy}$ at our observing frequency of $2.30 \mathrm{GHz}$. The adopted integrated flux density scale is thus that of Baars et al. (1977). As secondary calibrators (mainly used for pointing calibration) we chose the sources 3C 348, 3C 353, B0332-403, B0843-336, B1819-67, B1934-638 and B2356-61. 


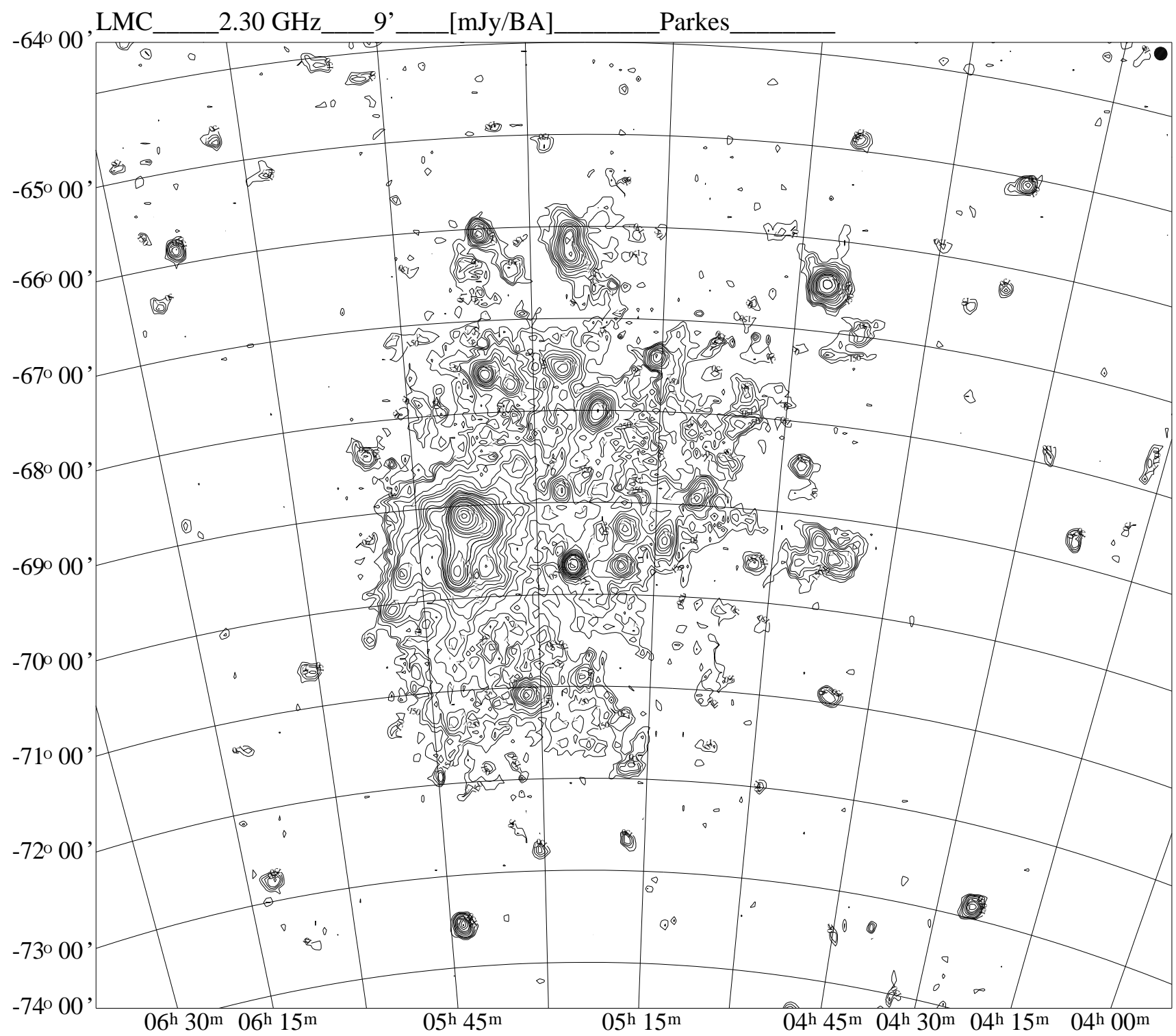

Fig. 1. The Parkes radio map of the $\mathrm{LMC}$ at $2.30-\mathrm{GHz}(\lambda=13 \mathrm{~cm})$. Contours are: $0.12,0.15,0.2,0.3, \ldots 1.0,1.5,2.0,3.0,5.0$, $7.5,10,15,20,25 \mathrm{Jy}_{\text {beam }}{ }^{-1}$. The observed beam size is $9^{\prime}$ and is shown in the upper right corner. One hundred and nineteen discrete sources were found in this survey (Table 1)

\section{Source fitting}

In the same way as in Paper IV, all data reduction for this study used the NOD2 software package (Haslam 1974), modified for the Parkes Telescope data. The automatic two-dimensional elliptical Gaussian-fitting algorithms implemented in the NOD2 package were used to search for sources and to determine source positions, flux densities and extension. A planar base level was fitted to the region of every source detected in the survey and the position and flux intensity of the discrete sources were then estimated. Sources were catalogued if their fitted Gaussian flux intensity was at least 5 times the rms noise level $(5 \sigma)$ in the image or if seen in at least one other different radio frequency survey. The validity of each source was then checked by inspecting the images and, for those regions in which there were clearly more than one discrete source, multi-source Gaussian fitting was used to model the extended emitting region. Because of the relatively large r.m.s. noise of this survey, we were unable to detect a number of weak sources $(\leq 150 \mathrm{mJy}$ at $2.30 \mathrm{GHz})$ from our 2.45 GHz survey (Paper IV, Table 3 ). 


\section{Radio source catalogue}

\subsection{The tables}

Table 1 is the catalogue of discrete radio sources found from this survey. This table is similar to Tables 2-6 from Paper IV. For the source name (Col. 1) we used the same convention we adopted in Paper IV. Source positions, right ascension and declination, are given in B1950 coordinates (Cols. 2 and 3). In Col. 4 the symbol (e) is used to flag sources that are significantly extended, as discussed below. The integrated flux densities are presented in Col. 5 .

Table 2 gives the cross-references for all sources in Table 1. Col. 3 (Other Names and Comments) uses the following abbreviations:

$N \quad$ Henize (1956) (e.g., N 157A),

$M C \quad$ McGee et al. (1972) (e.g., MC 74),

MC4 Clarke et al. (1976) (e.g., MC4(0539-691),

$D E M$ Davies et al. (1976) (e.g., DEM 263),

$L H G$ Long et al. (1981) (e.g., LHG 72),

$W \quad$ Wang et al. (1991) (e.g., W72),

NGC Sinnott (1988) (e.g., NGC 2070),

$P M N$ Wright et al. (1994)(e.g., PMNJ 0538-6905),

PSR Taylor et al. (1993) (e.g., PSR B0540-69),

LH Lucke \& Hodge (1970) (e.g., LH100),

Sa Sanduleak et al. (1978) and Sanduleak (1984)

(e.g., Sa 15),

$C O \quad$ Cohen et al. (1988) (e.g., CO 33),

PKS Otrupcek \& Wright (1991) (e.g., PKS B0539-691),

$I J L \quad$ Israel et al. (1993) (e.g., IJL 83), and

RASS Pietsch et al. (1996) (e.g., RASS 9008).

\subsection{Source extension}

The basket-weaving scanning process used during the observations slightly broadens the full width half maximum (FWHM) beam size. We have determined the effective beam size from the distribution of the fitted widths after excluding the tail of very extended sources. We excluded sources greater than 5 times the median size and than recalculated the median until the process converged ${ }^{1}$. The definition adopted here for a significantly extended source is that the fitted size of the source is twice that of the effective beam size. The true angular size of the sources is the observed size deconvolved with the effective beam size. Adopting this definition, approximately $40 \%$ (47 out of 119) of all the sources in this survey are extended. Table 3 gives details about nominal beam size, effective beam size, uncertainty in the effective beam size and the number of extended sources.

\footnotetext{
${ }^{1}$ The median rather then mean was used since the distribution was not Gaussian.
}

Table 1. Nominal and effective beam size, standard deviation of effective source size and number of sources considered as clearly extended

\begin{tabular}{ccccc}
\hline \hline $\begin{array}{c}\text { Frequency } \\
(\mathrm{GHz})\end{array}$ & $\begin{array}{c}\text { Nominal } \\
\text { Beam Size } \\
(\operatorname{arcmin})\end{array}$ & $\begin{array}{c}\text { Effective } \\
\text { Beam Size } \\
(\operatorname{arcmin})\end{array}$ & $\begin{array}{c}\text { Standard } \\
\text { Deviation } \\
(\operatorname{arcmin})\end{array}$ & $\begin{array}{c}\text { No. of } \\
\text { Extended } \\
\text { Sources }\end{array}$ \\
\hline 2.30 & 8.85 & 10.3 & 1.76 & 47 \\
\hline
\end{tabular}

\section{Calibration procedures}

\subsection{Position calibration and uncertainties in position}

The accuracy of the positions for all sources in our catalogue is limited by the pointing accuracy and beam size of the Parkes Telescope. We have compared positions of all 119 sources from this survey with the best available radio positions from other radio surveys (Paper IV) of the LMC (Fig. 2). The mean positional differences are satisfactory: $\Delta \mathrm{RA}=-3^{\prime \prime} \pm 4^{\prime \prime}, \Delta \mathrm{Dec}=-4^{\prime \prime} \pm 3^{\prime \prime}$ (best radio $-2.30 \mathrm{GHz}$ ). Thus, we have found no significant positional bias between our radio positions from this survey and those from Paper IV.

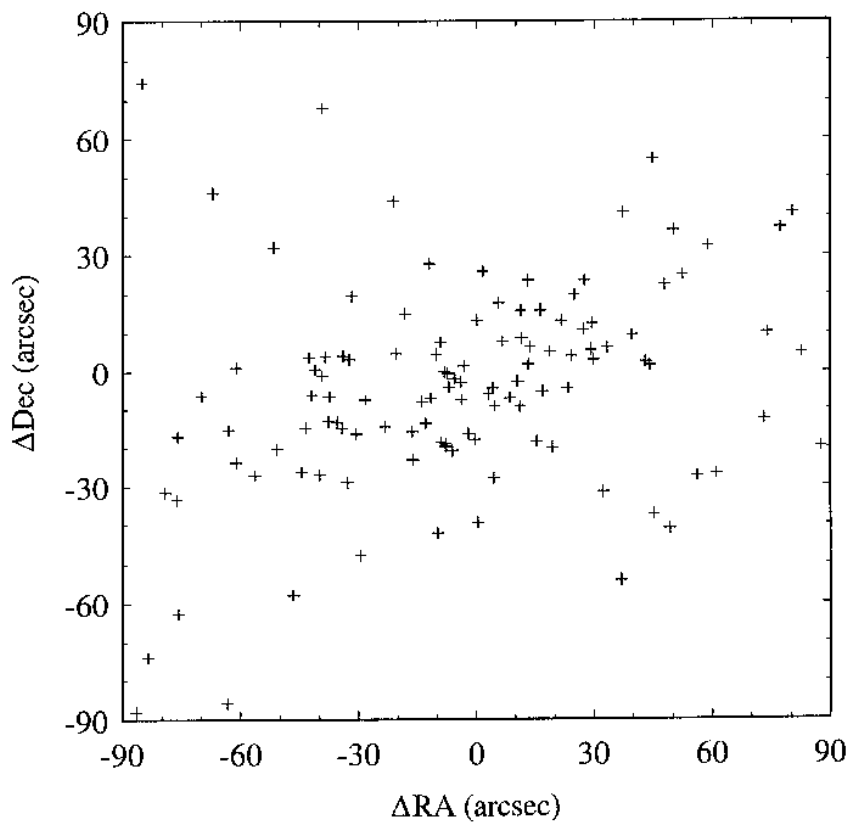

Fig. 2. Differences in positions between this $2.30 \mathrm{GHz}$ survey and best radio positions from Paper IV. The mean offset (Paper IV $-2.30-\mathrm{GHz}$ ) is $-3^{\prime \prime} \pm 4^{\prime \prime}$ and $-4^{\prime \prime} \pm 3^{\prime \prime}$ in RA and Dec, respectively. The standard deviations are $43^{\prime \prime}$ and $29^{\prime \prime}$, respectively

The standard deviations in the differences between the $2.30 \mathrm{GHz}$ data and the best radio positions are $43^{\prime \prime}$ in 
RA and $29^{\prime \prime}$ in Dec. These positional accuracies represent $\sim 10 \%$ of the Parkes Telescope beam size and are consistent with the combined positional uncertainties for the radio sources defined in Paper IV. However, the scatter in right ascension is slightly larger than in declination, as noted with the other Parkes surveys.

An additional comparison between our catalogues and accurate radio positions $\left(<0.5^{\prime \prime}\right)$ for two widely separated sources, B1934-638 and B0543-735, catalogued by White (1993), shows no significant positional bias.

\subsection{Flux density calibration and uncertainties in the flux densities}

\subsubsection{Comparison with the Parkes (PKSCAT-90) Catalogue}

To establish the relative flux density scales of our catalogue at $2.30 \mathrm{GHz}$ we have compared our flux densities with those from the PKSCAT-90 catalogue (Otrupcek \& Wright 1991) which includes sources from surveys at 2.70 GHz (McGee et al. 1972a; Bolton \& Butler 1975).

In Fig. 3a we show a comparison of the integrated flux density from our survey at $2.30 \mathrm{GHz}$ with the flux density at $2.70 \mathrm{GHz}$ (McGee et al. 1972a; Bolton \& Butler 1975 ) for 58 sources (excluding 30 Dor as it is very extended). The resulting mean ratio is $1.06 \pm 0.02$ (our flux densities are higher) with a scatter of $13.4 \%$. This is consistent with the expected ratio taking the mean spectral index $\alpha=-0.5$.

In Fig. 3b we show a similar comparison of the integrated flux density, $S$, of sources at $2.45 \mathrm{GHz}$ (Paper IV) with flux density at $2.30 \mathrm{GHz}$ from this work. A comparison of 112 of these sources observed by us show that the flux densities agree well. The mean ratio is $0.97 \pm 0.01$ with a standard deviation of $5.1 \%$.

In summary, the flux densities of our catalogue (Table 1) agree well with those of the catalogues used for comparison.

\subsubsection{Uncertainties in flux densities}

Following Paper IV, we have compared the flux densities at $2.30 \mathrm{GHz}$ with interpolated flux densities at the same frequency from PKSCAT-90 (Otrupcek \& Wright 1991). This type of analysis gives the uncertainty in flux density in the form:

$$
\Delta S=\sqrt{A^{2}+(B * S)^{2}}
$$

where $A$ and $B$ are constants. The flux-densityindependent component of uncertainty $(A)$ has been computed from the rms differences for weak sources (where flux-density-dependent uncertainties are negligible), and the flux-density-dependent uncertainties $(B)$ have been computed from the rms differences for strong sources. Assuming an equal rms noise for both our surveys and
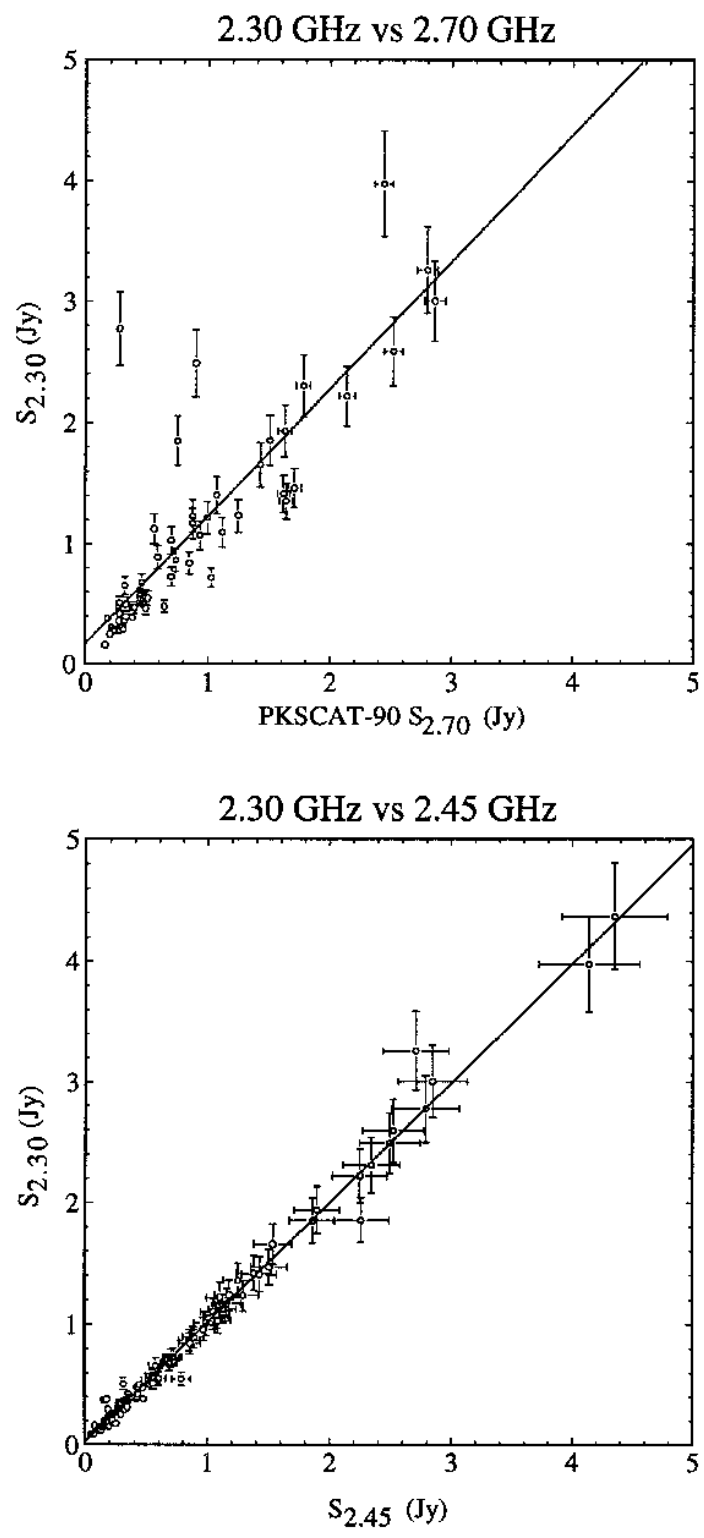

Fig. 3. Comparison of flux densities. In Fig. 3a we compare flux densities from Table 1 (measured at $2.30 \mathrm{GHz}$ ) with flux densities from PKSCAT-90 at $2.70 \mathrm{GHz}$. In Fig. 3b we compare flux densities from Table 1 (measured at $2.30 \mathrm{GHz}$ ) with flux densities from the $2.45 \mathrm{GHz}$ survey (Paper IV, Table 3)

the PKSCAT-90 compilation, we obtain $A=0.020$ Jy and $B=7.8 \%$. For all but the very weakest sources, the uncertainty in flux density is dominated by the flux-densitydependent component which is $7.8 \%$.

We note that this comparison with PKSCAT-90 yields a percentage uncertainty larger than that found for the inter-comparison of this work with the $2.45 \mathrm{GHz}$ catalogue of Paper IV. Therefore, we assume that the uncertainties in this catalogue and the $2.45 \mathrm{GHz}$ catalogue of Paper IV (Table 3 ) are probably overestimated by a factor of $\sim 2$. 


\section{Conclusions}

This work presents a new catalogue of 119 radio sources in the LMC at $2.30 \mathrm{GHz}$. For all of these sources we estimate positions and fluxes together with their uncertainties. Also, we have identified these sources at other wavelengths (other radio, optical, X-ray, etc.). Further analysis will appear in following papers, and will discuss the radio spectral indices and comparisons with X-ray, infrared and optical data.

Acknowledgements. We are very grateful to P. Reich, P. Müller and C. Sheerman for help with the NOD2 package and to M. Hunt, D.E. Goddard and R.A. Duncan for comments on the manuscript. M.D.F. acknowledges an Australian Government Overseas Postgraduate Research Scholarship and considerable support from the Australia Telescope National Facility (CSIRO) and Max-Planck-Institut für Radioastronomie (MPIfRA). This work is also supported by an Australian Research Council (ARC) research grant.

\section{References}

Baars J.W.M., Genzel R., Pauliny-Toth I.I.K., Witzel A., 1977, A\&A 61,99

Bolton J.G., Butler P.W., 1975, Aust. J. Phys. Astrophys. Suppl. 34, 33

Clarke J.N., Little A.G., Mills B.Y., 1976, Aust. J. Phys. Astrophys. Suppl. 40, 1

Cohen R., Dame T.M., Garay G., Montani J., et al., 1988, ApJ 331, L95

Davies R.D., Elliott K.H., Meaburn J., 1976, Mem. R. Astr. Soc. 81,89
Filipović M.D., Haynes R.F., White G.L., Jones P.A., et al., 1995, A\&AS 111, 1 (Paper IV)

Filipović M.D., Pietsch W. N., Haynes R.F., et al., 1996a, A\&A (in preparation)

Filipović M.D., White G.L., Haynes R.F., et al., 1996b, A\&A (in preparation)

Haslam C.G.T., 1974, A\&AS 15, 333

Haynes R.F., Klein U., Wielebinski R., Murray J.D., 1986, A\&A 159, 22

Haynes R.F., Klein U., Wayte S.R., et al., 1991, A\&A 252, 475

Henize K.G., 1956, ApJS 2, 315

Israel F.P., Johansson L.E.B., Lequeux J., et al., 1993, A\&A 276,25

Long K.S., Helfand D.J., Grabelsky D.A., 1981, ApJ 248, 925

Lucke P.B., Hodge P.W., 1970, AJ 75, 171

McGee R.X., Brooks J.W., Batchelor R.A., 1972a, Aust. J. Phys. 25, 613

McGee R.X., Brooks J.W., Batchelor R.A., 1972b, Aust. J. Phys. 25, 581

Otrupcek R.E., Wright A.E., 1991, Proc. Astron. Soc. Aust. 9, 170

Pietsch W., et al. , 1996 (in preparation)

Sanduleak N., MacConnell D.J., Davis Philip A.G., 1978, PASP 90, 621

Sanduleak N., 1984, In: Van den Bergh S., de Boer K.S. (eds.) Proc. IAU Symp. 108, Structure and Evolution of the Magellanic Clouds. Reidel, Dordrecht, p. 231

Sinnott R.W., NGC 2000, 1988, Cambridge Univ. Press, p. 46

Taylor J.H., Manchester R.N., Lyne A.G., 1993, ApJS 88, 529

Wang Q., Hamilton T., Helfand D.J., Wu X., 1991, ApJ 374, 475

White G.L., 1993, Proc. Astron. Soc. Aust. 8, 140

Wright A.E., Griffith M., Burke B., Ekers R.D., 1994, ApJS 91,111 